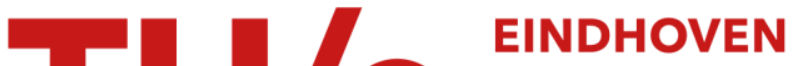

\section{Growth and chirality amplification in helical supramolecular polymers}

Citation for published version (APA):

Gestel, van, J. A. M., Schoot, van der, P. P. A. M., \& Michels, M. A. J. (2006). Growth and chirality amplification in helical supramolecular polymers. In R. G. Weiss, \& P. Terech (Eds.), Molecular gels : Materials with selfassembled fibrillar networks (pp. 79-97). Springer. https://doi.org/10.1007/1-4020-3689-2_3

DOI:

10.1007/1-4020-3689-2_3

Document status and date:

Published: 01/01/2006

\section{Document Version:}

Publisher's PDF, also known as Version of Record (includes final page, issue and volume numbers)

\section{Please check the document version of this publication:}

- A submitted manuscript is the version of the article upon submission and before peer-review. There can be important differences between the submitted version and the official published version of record. People interested in the research are advised to contact the author for the final version of the publication, or visit the $\mathrm{DOI}$ to the publisher's website.

- The final author version and the galley proof are versions of the publication after peer review.

- The final published version features the final layout of the paper including the volume, issue and page numbers.

Link to publication

\section{General rights}

Copyright and moral rights for the publications made accessible in the public portal are retained by the authors and/or other copyright owners and it is a condition of accessing publications that users recognise and abide by the legal requirements associated with these rights.

- Users may download and print one copy of any publication from the public portal for the purpose of private study or research.

- You may not further distribute the material or use it for any profit-making activity or commercial gain

- You may freely distribute the URL identifying the publication in the public portal.

If the publication is distributed under the terms of Article 25fa of the Dutch Copyright Act, indicated by the "Taverne" license above, please follow below link for the End User Agreement:

www.tue.nl/taverne

Take down policy

If you believe that this document breaches copyright please contact us at:

openaccess@tue.nl

providing details and we will investigate your claim. 


\section{Chapter 2}

\section{GROWTH AND CHIRALITY AMPLIFICATION IN HELICAL SUPRAMOLECULAR POLYMERS}

Jeroen van Gestel*, Paul van der Schoot, and M.A.J. Michels

Eindhoven Polymer Laboratories, Technische Universiteit Eindhoven, P.O. Box 513, 5600 MB Eindhoven, The Netherlands

$\begin{array}{ll}\text { 1. Introduction } & 79\end{array}$

2. Helical Aggregation 80

3. Discotics 82

4. Linear Self-Assembly $\quad 83$

5. A Two-State Model 85

6. Aggregate Ends 86

7. Chirality Amplification 90

8. Sergeants and Soldiers 91

9. Conclusions and Perspectives for the Future 94

\section{Introduction}

Helical self-assembled, supramolecular polymers have been known to exist in nature for some time [1-6]. It is well-established that aggregates such as f-actin [3, 4], tobacco mosaic virus [6] and $\beta$-sheet ribbons formed by certain oligopeptides [7] are helical. Recently, many different molecules have been synthesized that also self-assemble into helical conformations [8-16].

Helical self-assembly [17] can be a one-state or a two-state process, depending on the physical conditions. In the latter case, polymerization into non-helical aggregates precedes a conformational transition of the assemblies to a helical state. Both the helical transition and the polymerization can be triggered by

\footnotetext{
*Current address: Physical Chemistry and Molecular Thermodynamics Group, DelftChemTech, Julianalaan 136, 2628 BL, Delft, The Netherlands.
} 
changes in concentration or temperature $[18,19]$. Neither of the two are phase transitions in the true sense of the word, but more or less gradual crossovers. However, the helical transition in particular can be fairly sharp due to cooperative effects, leading to a remarkable temperature sensitivity. Because of this, it is possible to define the transition temperatures. See below.

Several of the known helical self-assembled systems seem to display a gellike phase at high enough concentrations, which is presumably linked with the helical transition $[3,8,10,20]$. The reason is that helical polymers are typically more rigid (i.e., have a larger radius of gyration) than non-helical ones, and that the helical transition is accompanied by a strong increase in the mean aggregate size and ultimately, when sufficiently long, entanglements.

\section{Helical Aggregation}

In order to understand the mechanisms driving the helical aggregation, a host of experimental and theoretical work has been done [3, 8-16, 21-23]. The most well-known theory in this context, is the Oosawa-Kasai treatment for the helical assembly of f-actin fibers from the g-actin protein [3, 23]. Due to their molecular architecture, the actin units are thought to be able to attach themselves to each other in two ways, one of which results in a non-helical assembly, and one in a helical one (see Figure 1). Oosawa and co-workers assign one equilibrium constant to each process $\left(K\right.$ and $\left.K_{h}\right)$. This means that their model is in essence an all-or-nothing model: partially helical polymers are completely suppressed. Similar models are still in use (see [24] and references cited therein).

In the Oosawa-Kasai model it is also possible for a non-helical chain to become helical or vice versa. This introduces another (generalized) equilibrium constant $\gamma\left(K_{h} / K\right)^{N-3}$ with $N>2$ the number of monomers in the aggregate, possibly linked to a conformational switching of the protein molecules. In this

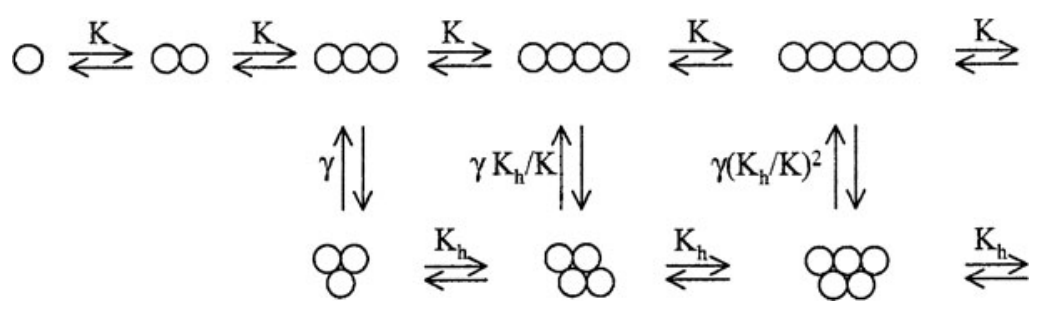

Figure 1. Schematic depiction of the Oosawa-Kasai multi-equilibrium model. $K$ is the equilibrium constant for the addition of a monomer in a non-helical conformation, $K_{h}$ is that for the addition in a helical conformation, and $\gamma$ is the nucleation parameter for a helical trimer [3]. 
picture, not every aggregate can undergo the transition from a non-helical to a helical state; for f-actin it is surmised that a critical nucleus of three monomeric units is necessary for helix formation. The model predicts that helical or nonhelical polymers form, dependent on the values of the three equilibrium constants. For $K_{h} \gg K$ and $\gamma \rightarrow 0$, a critical concentration for the formation of helical aggregates is required. All excess monomers above this concentration are absorbed into helical polymers that co-exist with the free monomers and with short non-helical polymers.

The theory of Oosawa and Kasai is easy to implement and can be expected to accurately describe systems requiring a nucleation step and which display a large co-operativity of the helical transition, because then the polymers become fully helical or fully non-helical. Indeed, the model seems to accurately describe the helical polymerization of f-actin [25]. However, when compared to experimental measurements on helical aggregates of disc-shaped monomers (discotics) [9, 21, 22], shown in Figure 2, the agreement is poor. See Figure 5, to be discussed in more detail below. Therefore, a different theoretical treatment is needed if we wish to describe helical supramolecular systems in general.

Here, we outline a recent extension $[21,22,26]$ of the theory of Oosawa and Kasai to polymers that need not be fully helical or non-helical. The wellknown Zimm-Bragg model for the helix-coil transition in polypeptides [27] is a similar improvement on the all-or-nothing model for this conformational transition in conventional polymers [18]. Our treatment accurately describes the conformational state of aggregates of the discotic molecules of Figure 2. The treatment is fairly general, however, and can, in principle, be applied to

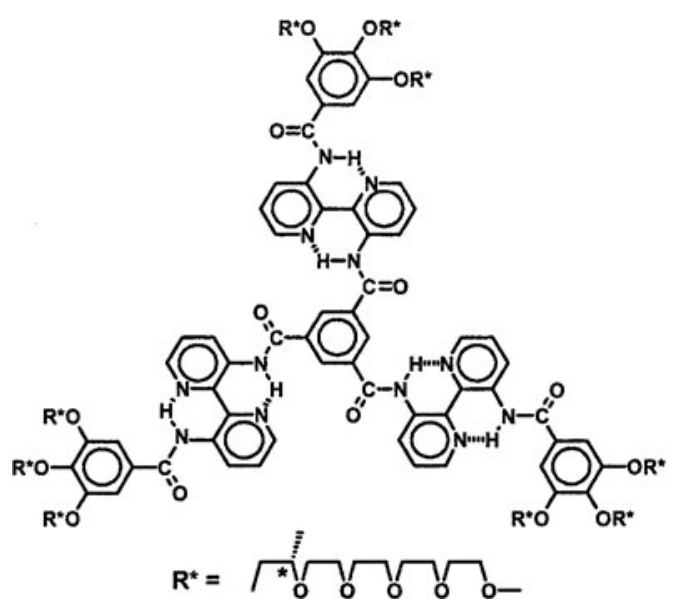

Figure 2. Chemical formula of the chiral discotic molecules used by Brunsveld and co-workers in their helical-assembly experiments [9]. The chemical name of the molecule is $\mathrm{N}, \mathrm{N}^{\prime}, \mathrm{N}$ "-tris $\{3[3$ '-(3,4,5-tris $\{(2 \mathrm{~S})-2-(2-\{2-[2-(2-$ methoxyethoxy)-ethoxy]ethoxy\}-ethoxy)-propyloxy\})-benzoylamino]-2,2'-bipyridyl \}benzene-1,3,5-tricarboxamide. 
any type of supramolecular polymer that displays a transition between a helical and a non-helical state.

Because the helical transition is accompanied by a sharp increase in the mean molecular weight of the supramolecular assemblies and that in their bending stiffness, the solution viscosity should also dramatically increase. Indeed, as is well known [28], the intrinsic viscosity of stiff, rod-like polymers grows with their molecular weight to the second power in dilute solution, and with a sixth power in semi-dilute solution when the rods overlap. This means that a tenfold increase in the molecular weight upon crossing the helical transition temperature could lead to a million-fold increase of the viscosity within a few degrees of temperature change. It is for this reason, that we believe that helical supramolecular polymers are promising candidates as gelating agents.

\section{Discotics}

The molecules we focus on in our comparison to experiment have a large aromatic core and nine flexible, polar, side chains (see Figure 2). This allows them to be dissolved in polar solvents, such as water and n-butanol. Since the core of the molecules is solvophobic, the monomers form stacks in solution [9]. From the experiments, it becomes clear that the monomers can form disordered linear aggregates, as well as helical aggregates with a relatively high degree of order (depending on the temperature and the concentration) [9, 29]. This can be explained microscopically by the propeller-like shape of the monomers, which allows for a stronger interaction if they take on a helical conformation by rotating the side groups out of the plane of the center of the monomer (see Figure 3) $[9,21]$. Phenomenologically, this corresponds to the situation we presumably have in actin assembly, in that both monomers have a molecular

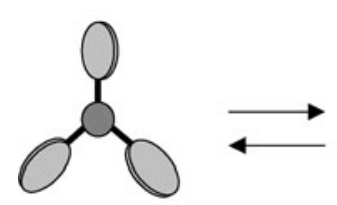

Monomer

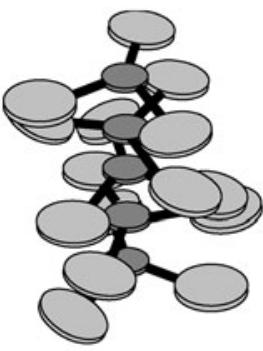

Weakly bound

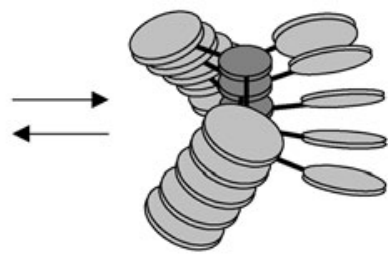

Strongly bound

Figure 3. Cartoon of the linear and helical aggregation of propeller-shaped discotic molecules. The molecules self-assemble into disordered stacks, in which they are more or less free to rotate. These stacks then undergo a transition to a more strongly bound, helical state, under the right conditions. 
architecture that accomodates two types of binding, and can therefore undergo a helical transition.

As shown in Figure 2, the discotics have a stereocenter in each of their side chains. In the experiments of Brunsveld and co-workers [9] only one of the enantiomers was used, which causes a bias for the formation of one helical screw sense. Such a bias may also follow from, e.g., the use of a homochiral solvent or homochiral counterions in ionic systems. If a racemic mixture of the enantiomers had been used, or an achiral discotic, equal amounts of righthanded and left-handed helices would form [30], and the conformational state of the polymers could not be determined by spectroscopic methods such as circular-dichroism (CD) spectroscopy. CD spectroscopy allows one to gauge the overall chirality in a solution, but it so happens that for the material in hand, it can be used to determine the helical state of the stacks because the individual monomers do not display a Cotton effect. See Chapter 13 for experimental details.

The disordered (non-helical) aggregates form due to the effects of mass action, whereby the monomers gain binding energy, but lose translational entropy relative to the free monomeric state. Upon lowering the temperature, the bound monomers decrease their configurational entropy further by taking on a helical conformation. This increases the binding enthalpy due to increased proximity between the monomers (see Figure 3 ). The addition of a non-helical bond to a helical aggregate or vice versa is unfavorable on steric grounds, as this would ultimately lead to a frustrated conformational state. Nonetheless, unfavorable or not, such thermally excited states would form for entropic reasons, and are the reason why the theory of Oosawa and Kasai needs to be amended.

\section{Linear Self-Assembly}

Let us first focus on the theory of linear self-assembly (i.e., the self-assembly of monomeric units into polymer-like chains) in which these monomers are connected to each other by physical, reversible bonds [24, 31-37]. There are many ways to theoretically deal with linear self-assembly [36, 38-47]. In the simplest Ansatz, the aggregation depends solely on a single energetic parameter, describing the free energy expended to break an aggregate into two. This free energy cost is assumed to be independent of the point along the polymer where the break is introduced [48, 49]. It is known in the field of giant micelles [48] as the scission energy or the end-cap energy. For supramolecular polymers which can undergo a helical transition, a single free-energy parameter does not necessarily suffice, because the aggregate ends can have two different conformations, and hence different energies [22, 26]. Also, and perhaps more importantly, the chain becomes subject to conformational fluctuations that cannot be described by a scission energy alone. 
Generally, the dimensionless grand potential density $\Omega$ of a solution of non-interacting aggregates can be written as:

$$
\Omega=\sum_{N=1}^{\infty} \rho(N)[\ln \rho(N)-1-\mu N-\ln \Xi(N)]
$$

with $\rho(N)$ the (dimensionless) number density of aggregates of degree of polymerization $N, \mu$ the chemical potential of the monomers, and $\Xi(N)$ the generalized partition function of an individual aggregate. Note that all energies are given here in units $k_{B} T$, with $k_{B}$ Boltzmann's constant and $T$ the absolute temperature, unless otherwise specified. By setting $\delta \Omega / \delta \rho(N)=0$, we find for the equilibrium size distribution:

$$
\rho(N)=\Xi(N) \exp \mu N
$$

It is obvious from Eq. (2) that any terms in the free energy $-\ln \Xi(N)$ of a single chain, that are extensive (i.e., proportional to $N$ ) can simply be absorbed into the chemical potential $[48,50]$. This means that non-extensive terms in $\ln \Xi(N)$, which are often ignored altogether in the context of conventional polymers, are crucial to the description of equilibrium polymers and in fact explain the emergence of the scission energy alluded to in the beginning of this section. We cannot neglect them here as they couple directly to the size distribution of the equilibrium polymers.

We can fix the chemical potential, $\mu$, by invoking conservation of mass (Eq. (3)).

$$
\phi=\sum_{N=1}^{\infty} N \rho(N)
$$

Here, $\phi$ is the volume fraction of self-assembling material in the solution. The mean size of the aggregates may be found from Eq. (4).

$$
\langle N\rangle \equiv \frac{\phi}{\sum_{N=1}^{\infty} \rho(N)}
$$

The crucial ingredient in the theory that contains all the information about the conformational state of the aggregates is the partition function $\Xi(N)$. It follows from Eq. (2) that the size distribution of the aggregates and their conformational state are closely linked. If only one type of bond is formed, the partition function effectively takes the form $\Xi(N)=\exp (N-1) E$, with $-E$ the free energy of the formation of a bond. (This is true irrespective of the chain model; it holds for rods and flexible chains alike.) The mean aggregate size is then expressed by Eq. (5).

$$
\langle N\rangle=\frac{1}{2}+\frac{1}{2} \sqrt{1+4 \phi \exp E}
$$


If two types of bonds are allowed, the situation is slightly more involved, as is detailed in the next section.

\section{A Two-State Model}

While the conformational state of polymers depends on many factors, such as the molecular architecture and the solvent composition, the helix-coil transition of polypeptides and other helix-forming polymers can be accurately described in terms of a simple quasi one-dimensional model known as the Zimm-Bragg model $[18,27]$. It concerns a two-state model that can be mapped onto the Ising chain $[18,51-53]$. "Interfaces" between helical and non-helical regions along the aggregate axis are attributed a free-energy penalty denoted $R$ that is related to the coupling constant in the Ising model.

Within a slight modification of the Zimm-Bragg theory, necessary to apply it to equilibrium polymers, the partition function becomes Eq. (6) [21, 22, 27].

$$
\Xi(N)=\left[x \lambda_{+}^{N-2}+y \lambda_{-}^{N-2}\right] \exp E(N-1)
$$

Here, $-E$ is the free energy of a non-helical bond between two neighboring monomers, and the weights $x$ and $y$ represent non-extensive contributions to the free energy that depend on the boundary conditions imposed on the aggregate ends (see below) [26]. The quantities $\lambda_{+}$and $\lambda_{-}$are the eigenvalues of the so-called transfer matrix of the Ising chain [54]. For a detailed description of the transfer matrix method we refer to the book of Poland and Scheraga [18]. From the Zimm-Bragg theory we have Eq. (7).

$$
\lambda_{ \pm}=\frac{1}{2}+\frac{1}{2} s \pm \frac{1}{2} \sqrt{(1-s)^{2}+4 \sigma s}
$$

$s \equiv \exp -P$, where $P$ denoted the excess free energy of a helical bond over a non-helical one, and $\sigma \equiv \exp -2 R$ the square of the Boltzmann factor of the free energy penalty $R$ imposed on an interface between a helical and non-helical part of the chain. The latter is often seen as a co-operativity parameter, because the larger $R$ becomes, the fewer interfaces form, and the longer the helical and non-helical regions become $[27,55]$. This implies a large co-operativity for small values of $\sigma$.

Since $\lambda_{-}$is always smaller than $\lambda_{+}$, the second term of Eq. (6) is usually neglected in the limit where $N \gg 1$; this is the so-called ground-state approximation. It corresponds to the earlier-mentioned treatment of ideal linear aggregates, albeit with a renormalized scission energy. Often, however, the aggregates turn out to be too small to be accurately described with a ground-state theory, and the full description as in Eq. (6) is required, a fact underappreciated by workers in the field of giant micelles. 
Note that Eq. (6) is only valid for $N \geq 3$. For monomers we set $\Xi(1)=1$, whereas for dimers, the partition function depends on the boundary conditions we enforce and becomes $\Xi(2)=x+y$. The importance of the choice of boundary conditions is discussed in more detail in the next section.

The mean fraction of helical bonds in a single aggregate can be calculated from Eq. (8).

$$
\langle\theta(N)\rangle \equiv \frac{1}{(N-1)} \cdot \frac{\partial \ln \Xi(N)}{\partial \ln s}
$$

In the limit $N \rightarrow \infty$ we have $\langle\theta(N)\rangle \sim \frac{1}{2}+\frac{1}{2}(s-1) / 2 \sqrt{(s-1)^{2}+4 s \sigma}$. At the helical-transition temperature, $T_{* *}$, we require that half the bonds are helical, so that $\langle\theta(N)\rangle=1 / 2$, i.e., for infinitely long chains it is given by $s=1$. If we average over all aggregate sizes, we get Eq. (9).

$$
\langle\theta\rangle \equiv \frac{1}{\phi-\rho(1)} \sum_{N=2}^{\infty} N \rho(N)\langle\theta(N)\rangle
$$

\section{Aggregate Ends}

It seems reasonable to suggest that the conformational state of monomers near the ends of the chains are different from those in the center, because the local environments of the monomers are different. Some care must be taken in the description of the ends of the aggregates, because they have a large impact on the self-assembly of the chains, as noted in Section (4) [26]. To account for this, we define the Boltzmann factors $a, b, c$ and $d$ for helical and non-helical bonds at either aggregate end, where $a$ and $c$ represent a non-helical end, and $b$ and $d$ a helical one. (Note that the ends of an aggregate need not be identical since the helix is an object with an inherent direction.) The prefactors of Eq. (6) then become Eqs. (10) and (11).

$$
\begin{aligned}
& x=\frac{\left(a \sqrt{\sigma}+b \lambda_{+}-b\right)\left(c-c \lambda_{-}+d s \sqrt{\sigma}\right)}{\sqrt{\sigma}\left(\lambda_{+}-\lambda_{-}\right)} \\
& y=\frac{\left(a \sqrt{\sigma}+b \lambda_{-}-b\right)\left(c \lambda_{+}-c-d s \sqrt{\sigma}\right)}{\sqrt{\sigma}\left(\lambda_{+}-\lambda_{-}\right)}
\end{aligned}
$$

Even in the ground-state approximation, the aggregate ends influence the scission energy, which becomes $E+2 \ln \lambda_{+}-\ln x$, and not $E$. Interestingly, depending on the properties of the aggregate ends, the polymerization may in fact require a nucleation step. For instance, if we consider the case that one of the aggregate ends must be non-helical (so $a=b=c=1, d=0$ ), we must automatically assume that all dimers are also non-helical [26]. If we are in the regime where the helical bond is more favorable than the non-helical one 
$(s>1)$, the aggregates become helical as they grow. This means that the nonhelical short chains represent a nucleation barrier, since, in order to form a long helical chain, a non-helical (less energetically favorable) nucleus must first be formed. This recalls the cases of actin polymerization and $\beta$-sheet tape formation, in which nucleation is also believed to be required [3, 7].

Since the state of the ends couples strongly to the self-assembly, and since the self-assembly couples to the helical state of the assemblies, the ends in fact influence the nature of the helical transition in a non-trivial way. This is shown in Figure 4. We obtain good fits to experimental data only if we let at least one of the ends be helical. The figure also shows that the transition from a non-helical aggregate to a helical one must be highly cooperative, since the curve is quite steep at the transition temperature $T_{* *}$. From the steepness of the curve at this point, the Boltzmann factor for the formation of an "interface", $\sqrt{\sigma}$, may be obtained, as in Eq. (12), provided the enthalpy $\Delta h$ for the helical transition is known [21].

$$
\sqrt{\sigma} \Delta h=\left.4 T_{* *} \frac{\partial\langle\theta\rangle}{\partial T}\right|_{T_{* *}}
$$

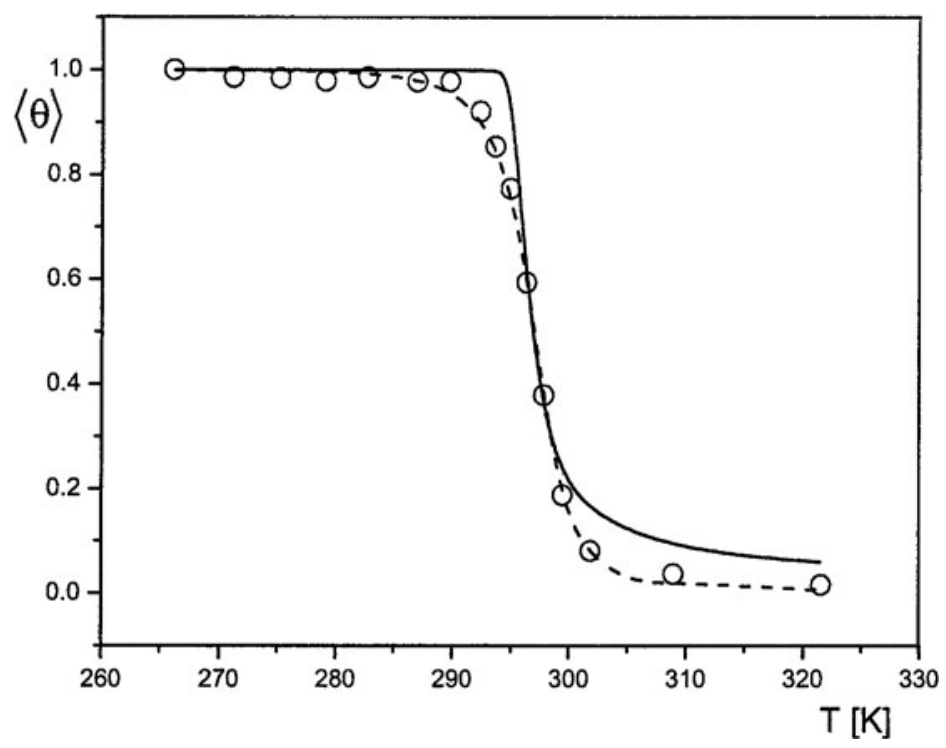

Figure 4. The fraction of helical bonds as a function of temperature. The symbols indicate experimental circular-dichroism data on solutions in n-butanol of the discotics of Figure 2, at a volume fraction of monomers of $2.55 \cdot 10^{-4}$ [9]. The lines give the best theoretical agreement with experiment, given the boundary conditions imposed. Drawn line: boundary conditions one end helical. Dashed line: one end non-helical. The helical-transition temperature, $T_{* *}$ (where $\langle\theta\rangle=1 / 2)$ is approximately equal to $296 \mathrm{~K}$. 
For the material of Figure 2 dissolved in n-butanol, we find a value of the Zimm-Bragg parameter $\sigma=0.0015$ (indicating a relatively high cooperativity, comparable to that in some covalently bound helical polymers [18]) when we insert the value for the excess helical-bonding enthalpy of $-27\left(k_{B} T\right)$, obtained by differential scanning calorimetry measurements [9].

A comparison between experimental data and theoretical predictions on the concentration dependence of the helical-transition temperature is shown in Figure 5, highlighting again the importance of a careful description of the ends. We find that the experimental system at hand can be well-described by the limiting case conditions where we presume one or both ends to be non-helical ( $a=b=c=1, d=0$ ). Both the Oosawa-Kasai model and our model with both ends free (as well as those with one or both ends helical - results not shown) describe the experimental results poorly. For the latter set of boundary conditions, this is because a helicity of one-half can always be attained, irrespective of the size of the aggregates, whereas with the other boundary conditions the dimers (and trimers) can never have a different conformation than that enforced by the boundary conditions.

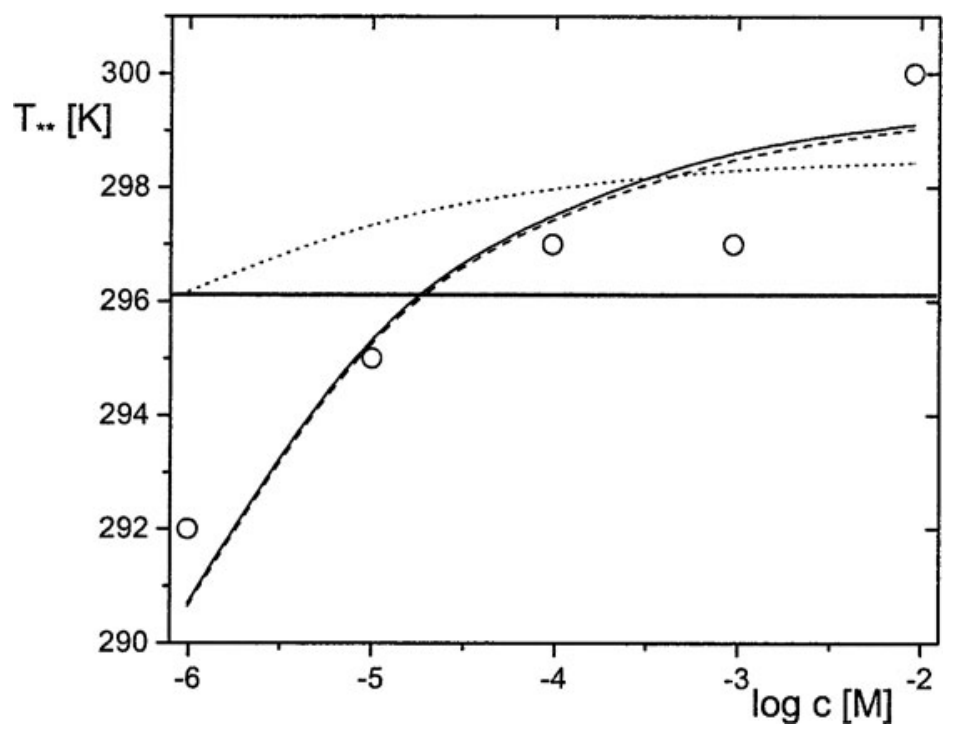

Figure 5. The helical-transition temperature $T_{* *}$ in $\mathrm{K}$ versus the concentration in $\mathrm{M}$ of the discotic compound of Figure 2 in n-butanol [9]. The symbols represent the experimentally found values, the curves give the theoretical results for different limiting boundary conditions: the dashed curve gives the results of the case with one end free and one fixed non-helical ( $a=b=c=1, d=0$ ), the drawn curve that for the case with both ends non-helical ( $a=c=$ $1, b=d=0$ ), and the thick (horizontal) line that for the case where both ends are left free ( $a=b=c=d=1)$. The dotted curve is a fit to the Oosawa-Kasai model [3, 23]. 
Focusing on the imposed boundary conditions that best describe the experimental data (i.e., with one end non-helical), we compare small angle neutron scattering (SANS) measurements to the mean aggregate size in Figure 6, and obtain reasonable agreement. If the temperature drops below the helicaltransition temperature (indicated by an arrow in Figure 6), the mean size of the aggregates exhibits a growth spurt. It shows that there is a strong coupling between the internal conformational state of the polymers and their growth. Unfortunately, given the experimental data of Figure 6, we cannot distinguish between the only two boundary conditions that describe the helical transition in the discotics well, although in principle it should be possible to distinguish them by means of the mean aggregate size measured for a larger temperature range (see the inset of Figure 6).

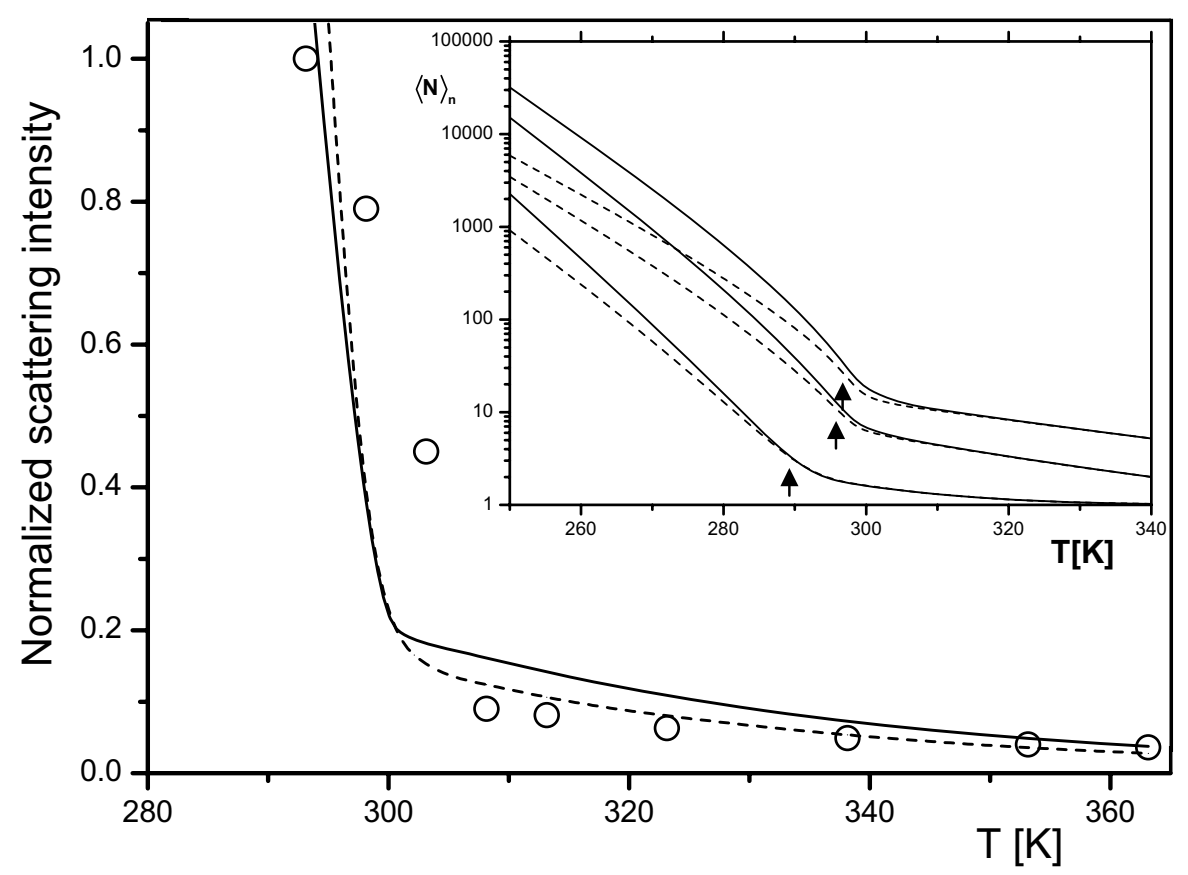

Figure 6. Theoretical fits to the results of SANS measurements on the system of the discotic molecules depicted in Figure 2 in n-butanol at a concentration of $2.39 \cdot 10^{-3} \mathrm{M}$. On the vertical axis is the normalised scattering intensity (which is a measure for the mean aggregate size), on the horizontal axis the temperature in $\mathrm{K}$. The drawn line gives the weight-averaged aggregate size as a function of the temperature, rescaled to give the best possible fit, for the limiting case with one end free and one non-helical $(a=b=c=1, d=0)$. The dashed line gives the same for the case with both ends non-helical $(a=c=1, b=d=0)$. In the inset is the mean aggregate size as a function of the temperature, for the concentrations (from top to bottom) $9.21 \cdot 10^{-3} \mathrm{M}, 9.64 \cdot 10^{-5} \mathrm{M}$ and $9.89 \cdot 10^{-7} \mathrm{M}$, with the dashed line the case with one end non-helical and the drawn line that with both ends fixed to be non-helical [21]. 


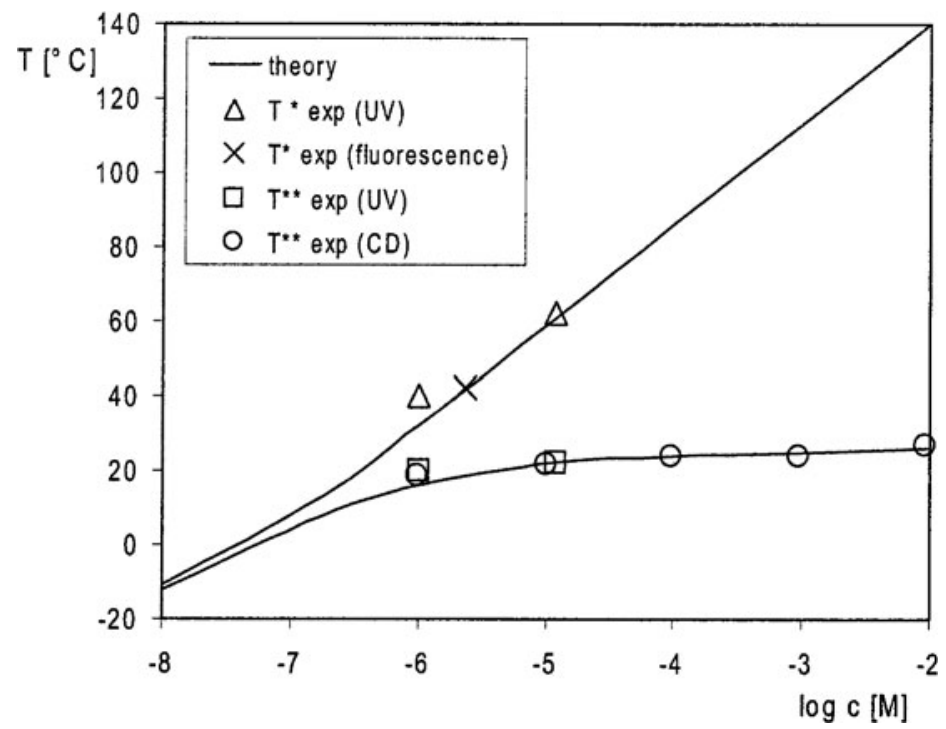

Figure 7. Diagram of aggregated states of the discotic monomers (see Figure 2) in n-butanol in terms of the temperature in ${ }^{\circ} \mathrm{C}$ and solute concentration in $\mathrm{M}$. The symbols indicate experimental results obtained with different techniques, such as circular-dichroism spectroscopy (CD), timeresolved fluorescence spectroscopy, and UV spectroscopy, as indicated. The lines indicate the temperatures where the theory predicts the helical (bottom, $T_{* *}$ ) and polymerization (top, $T_{*}$ ) transitions to take place. At $T_{* *}$ half the bonds are helical, at $T_{*}$ half the dissolved material is absorbed into aggregates [9]. Hence, the regime at the bottom of the Figure is the helicalchain regime, in between the two curves non-helical chains dominate, and the top regime is the monomeric regime.

In Figure 7 we summarise our results with a diagram of states. It would seem that our model captures the essential physics of the problem at hand. We conclude that there are two regimes, one where the polymerization and helical transitions are far apart, and one where they practically coincide. Here, the crossover from one to the other is regulated by the concentration of aggregating material. A similar shift may be induced by a change in solvent $[9,56,57]$.

\section{Chirality Amplification}

Another phenomenon of interest observed in supramolecular polymers is chirality amplification [30, 58-61]. It is linked with the presence of both leftand right-handed helical conformations, either in different assemblies or within a single assembly; if the monomers are achiral, or if racemic mixtures of chiral ones are present in the solution, both helical screw senses are formed in equal amounts. The two forms are mirror images of each other, and one can therefore 
say that the formation of a helix introduces a macromolecular chirality into the system [62]. Clearly, macromolecular (or in this case, supramolecular) chirality cannot easily be detected if there is no net molecular chirality. Symmetry can be broken by polymerizing homochiral material, leading to a bias for one of the helical screw senses.

Often, however, the copolymerization of only a small amount of chiral material suffices to introduce a bias for one of the helical screw senses, a phenomenon referred to in the field as chirality amplification. Two types of chirality amplification can be distinguished: the "sergeants-and-soldiers" type [58-60, 63], and the "majority-rules" type [61, 64]. The former occurs if a small amount of homochiral material is mixed with a large amount of achiral monomers. A large number of bonds with the screw sense preferred by the chiral monomers is then formed. The majority-rules principle is similar, except that the monomers used are the enantiomeric forms of the (chiral) monomers. A slight majority of one of the enantiomers produces a disproportionately large preference for one screw sense.

\section{Sergeants and Soldiers}

Experimentally, chirality amplification of the sergeants-and-soldiers type has been observed in solutions of mixtures of chiral and achiral discotics $[60,65,66]$. Again, two regimes are found, one where the effect is independent of the concentration of dissolved material, and one where this is not so. That a treatment quite similar to that of the Zimm-Bragg theory described in Section 5, is able to fit the experimental data in both regimes can be clearly seen from Figures 8 and 9 [67, 68]. Perhaps the most striking feature of the Figures is the maximum that occurs. How we deal with this feature theoretically is described in some detail below. (For a similar treatment on the majority-rules principle the reader is referred to the literature [69].)

The adaptation of the theory of Section 5 to model supramolecular chirality amplification is fairly straightforward, with right-handed and left-handed helical bonds replacing the helical and non-helical bonds. This means that $s$ now gives the Boltzmann factor of a right-handed helical bond over a left-handed one, and $\sigma$ is the square of the Boltzmann factor of a helix reversal [67]. The analogy is not exact, however, since in chirality amplification two types of monomer are present (chiral and achiral in the sergeants-and-soldiers experiment and left-handed and right-handed for the majority-rules principle), requiring an additional chemical potential. Another (obvious) small difference is in the interpretation of experimental results, where the measured optical effect corresponds to the difference between the fractions of right- and left-handed helical bonds, rather than the bare number of helical bonds. This means we study a mean net helicity $\eta \equiv 2\langle\theta\rangle-1$ rather than the mean bare helicity $\langle\theta\rangle$. 


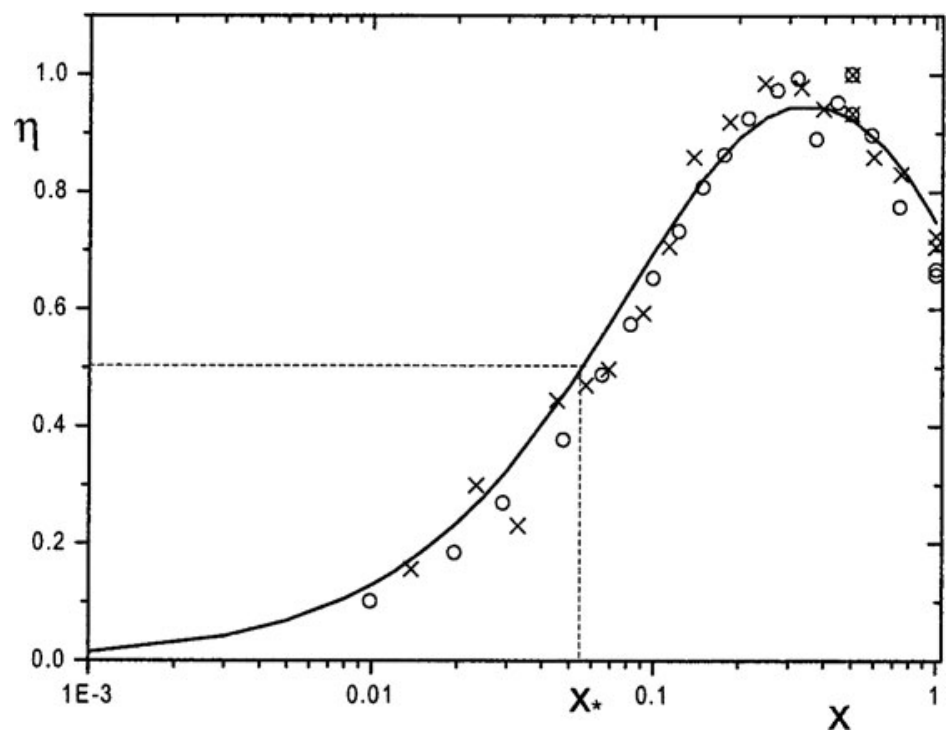

Figure 8. Sergeants-and-soldiers experiment. The net helicity $\eta$ versus the fraction of chiral material $x$ (line). Symbols: experimental data of Brunsveld et al. in water at two concentrations [65]. (Circles: $10^{-5} \mathrm{M}$, crosses $10^{-4} \mathrm{M}$ ). Line: fitted theoretical curve.

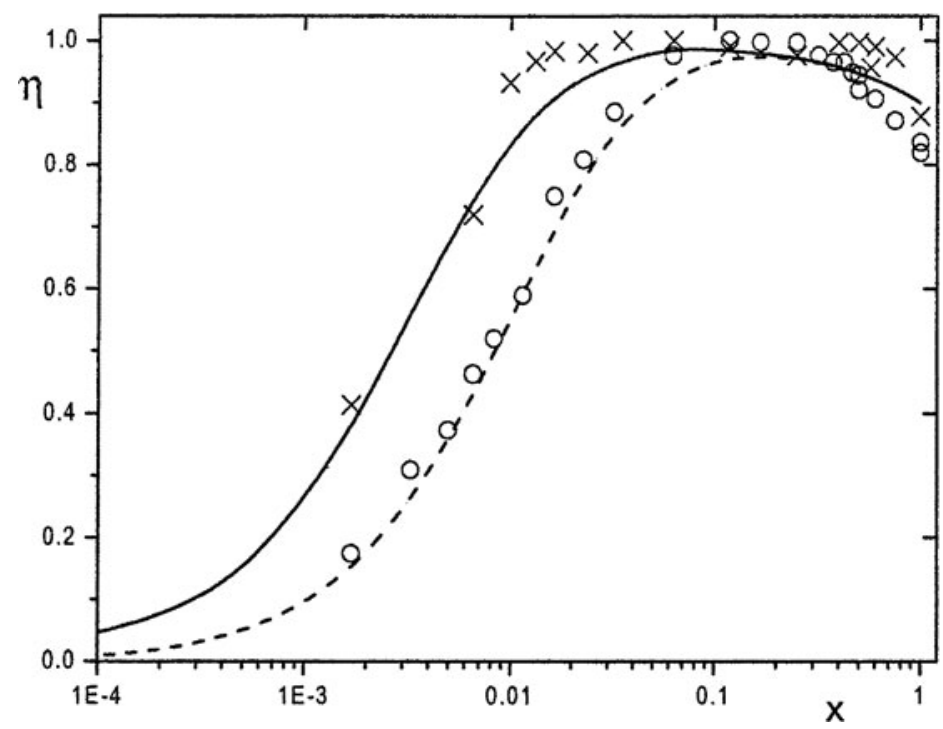

Figure 9. Fit of the theoretical net helicity $\eta$ versus $x$ to experimental data of Brunsveld et al. [66] in n-butanol at two concentrations. Circles: $10^{-5} \mathrm{M}$, crosses $10^{-4} \mathrm{M}$. Dashed line: fit for $10^{-5} \mathrm{M}$. Solid line: fit for $10^{-4} \mathrm{M}$. 
To account for the extra component, the Boltzmann weight $s$ of Section 5 has to be replaced by $1+z$, with $z$ the excess fugacity of the chiral monomers over the achiral ones. Note that there is no a priori preference for left- or right-handed helical bonds in achiral aggregates. In order to keep the number of free parameters to a bare minimum, we insist that a bond following a chiral monomer must always be right-handed helical, mimicking an absolute preference of the chiral units for a certain screw sense.

That our treatment is reasonable is shown in Figure 8, where we compare theoretical and experimental values for the net helicity $\eta$ versus the fraction of homochiral monomers $x$, and obtain very good agreement. In the limit of large cooperativity (where the Boltzmann factor of a helix reversal, $\sqrt{\sigma}$, is much smaller than unity) we find that the point $x_{*}$ at which the net helicity is one-half of its maximum value (Eq. (13)).

$$
x_{*} \approx \frac{1}{2} \sqrt{3 \sigma}
$$

It provides an easy way to determine the helix-reversal penalty from experiment. A qualitative argument that immediately gives Eq. (13) is that one in principle only needs one chiral monomer per correlation length to get a fully right-handed helical chain, and that the correlation length scales with $\sigma^{-1 / 2}$. (This follows from the Ising model.) Note that both Eq. (13) and Figure 8 are independent of the concentration of dissolved material.

In Figure 9, we give results similar to those in Figure 8, except that the chirality amplification now does depend on the concentration. We interpret the concentration dependence as an aggregate-size dependence, since in selfassembly the concentration and aggregate size are well known to be linked (see Eq. (5)). This means that we can describe the concentration-independent results of Figure 8 by assuming long chains (and implementing the ground-state approximation). In this case, the size of the aggregates is not an important factor, and the conformational state of the aggregates can be described in terms of the free energy of a helix reversal. Conversely, in the concentration-dependent (short-chain) regime of Figure 9, the aggregates are too short to contain many helix reversals, and in this case the average size of the aggregates determines how many bonds a single chiral monomer influences. The amount of chiral material needed to fully bias the handedness of an average chain is given by the number of chains in the solution. A crude estimate for $x_{*}$ is therefore given by Eq. (14) where $\langle N\rangle$ is given by Eq. (5).

$$
x_{*} \approx \frac{1}{2\langle N\rangle}
$$

On average half the chains then have a fixed handedness. The full theory gives a somewhat better estimate than Eq. (14), but needs to be evaluated numerically.

To fit the theory to the concentration-independent case (Figure 8) we extract an estimate of the parameter $\sigma$ at the point $x_{*}$ using Eq. (13), and fix it at 
$\sigma=0.0064$. We describe the maximum in the optical activity by taking into account the possibility of different contributions to the overall optical effect of chiral and achiral monomers. This may be due to imperfect stacking, as suggested by Brunsveld and co-workers [65, 67], since the chiral discotic has an extra methyl group in each of its side chains and therefore potentially attains a less perfect helix than the achiral monomer. This means that the optical rotation per monomer may vary between the species. We assume that the chiral monomers have a lower contribution to the overall optical effect than the achiral ones, and so obtain a maximum in the curve. A detailed discussion of this is beyond the scope of this chapter. We therefore refer to earlier work $[67,68]$.

For the concentration-dependent data of Figure 9, we perform a fit with the parameter $\phi \exp E$ to fix $x_{*}$ using Eq. (14) (see Section 4). We obtain good agreement with the experimental results at the higher concentration for a value of this parameter of 60000 . The bottom curve corresponds to a concentration of a factor ten lower than the top one, and as such should give good agreement for $\phi \exp E=6000$. This is indeed the case, demonstrating that our model takes the concentration dependence into account in a proper way (see Figure 9). Note that the chirality amplification becomes independent of $\sigma$ in this regime.

\section{Conclusions and Perspectives for the Future}

The complex behaviour of monomeric units that can undergo helical self-assembly can be captured by a relatively simple theory. It allows for the (quantitative) prediction of such quantities as the fraction of helical bonds (observable by circular-dichroism spectroscopy) and the mean aggregate size (measurable by radiation scattering techniques). We find that the aggregate ends have a large influence on the conformational state of the monomers inside the aggregates, and that it can be determined what boundary conditions apply to a specific system by comparing our theory to the experimental results.

Experiment and theory point at the presence of two regimes: one where the helical transition and the polymerization are two separate transitions, and one where they coincide. Which regime a system finds itself in depends on the concentration of monomers and on the solvent type. As is also the case in many helix-forming covalently-bound polymers, the helical transition is strongly cooperative and an aggregate can become helical due to a change in temperature of only a few degrees. Furthermore, it appears (experimentally and theoretically) that the polymerization and the helical transition are coupled; the polymers exhibit a growth spurt below the helical-transition temperature.

With some modifications, our model can also be used to describe chirality amplification in self-assembled helical polymers. Here, too, we find two regimes, in accord with experiments. In one regime, the strength of the chirality amplification depends on the concentration of self-assembling material, and 
in the other it does not. In each regime a different control parameter dictates the conformational state of the polymers. In the concentration-independent (long-chain) regime, this is the free energy of a helix reversal along the chain, in the concentration-dependent (short-chain) regime, it is the mean aggregate size.

Extensions of our treatment to cases where the aggregates interact, form liquid-crystalline phases or require an activation step before self-assembly seem worthwhile. Work on applying our model to the majority-rules type of chirality amplification and comparison to experimental results is in progress $[69,70]$.

\section{References}

[1] Friedhoff, P.; von Bergen, M.; Mandelkow, E.-M.; Davies, P.; Mandelkow, E. Proc. Natl. Acad. Sci. USA, 1998, 95, 15712.

[2] Watts, N.R.; Misra, M.; Wingfield, P.T.; Stahl, S.J.; Cheng, N.; Trus, B.L.; Steven, A.C.; Williams, R.W. J. Struct. Biol., 1998, 121, 41.

[3] Oosawa, F.; Asakura, S. Thermodynamics of the Polymerization of Protein, 1st edition, London: Academic Press, 1975.

[4] Oosawa, F. Biophys. Chem., 1993, 47, 101.

[5] Korn, E.D. Physiol. Rev., 1982, 62, 672.

[6] Klug, A. Angew. Chem. Int. Ed. Engl., 1983, 22, 565.

[7] Aggeli, A.; Nyrkova, I.A.; Bell, M.; Harding, R.; Carrick, L.; McLeish, T.C.; Semenov, A.N.; Boden, N. Proc. Natl. Acad. Sci. USA, 2001, 98, 11857.

[8] Brunsveld, L.; Folmer, B.J.B.; Meijer, E.W. MRS Bulletin, 2000, 25, 49.

[9] Brunsveld, L.; Zhang, H.; Glasbeek, M.; Vekemans, J.A.J.M.; Meijer, E.W. J. Am. Chem. Soc., 2000, 122, 6175.

[10] Hirschberg, J.H.K.K.; Brunsveld, L.; Ramzi, A.; Vekemans, J.A.J.M.; Sijbesma, R.P.; Meijer, E.W. Nature, 2000, 407, 167.

[11] Van Nostrum, C.F.; Bosman, A.W.; Gelinck, G.H.; Schouten, P.G.; Warman, J.M.; Kentgens, A.P.M.; Devillers, M.A.C.; Meijerink, A.; Picken, S.J.; Sohling, U.; Schouten, A.-J.; Nolte, R.J.M. Chem. Eur. J., 1995, 1, 171.

[12] Yoshida, N.; Harata, K.; Inoue, T.; Ito, N.; Ichikawa, K. Supramol. Chem., 1998, 10, 63.

[13] Gallivan, J.P.; Schuster, G.B. J. Org. Chem., 1995, 60, 2423.

[14] Lovinger, A.J.; Nuckolls, C.; Katz, T.J. J. Am. Chem. Soc., 1998, 120, 264.

[15] Fenniri, H.; Mathivanan, P.; Vidale, K.L.; Sherman, D.M.; Hallenga, K.; Wood, K.V.; Stowell, J.G. J. Am. Chem. Soc., 2001, 123, 3854.

[16] Engelkamp, H.; Middelbeek, S.; Nolte, R.J.M. Science, 1999, 284, 785.

[17] van Gestel, J. PhD thesis, Technische Universiteit Eindhoven, 2003.

[18] Poland, D.; Scheraga, H.A. Theory of Helix-Coil Transitions in Biopolymers, New York: Academic Press, 1970.

[19] Bloomfield, V.A. Am. J. Phys., 1999, 67, 1212.

[20] MacKintosh, F.C.; Janmey, P.A. Curr. Opin. Solid State Mater. Sci., 1997, 2, 350.

[21] van der Schoot, P.; Michels, M.A.J.; Brunsveld, L.; Sijbesma, R.P.; Ramzi, A. Langmuir, 2000, 16, 10076.

[22] van Gestel, J.; van der Schoot, P.; Michels, M.A.J. J. Phys. Chem. B, 2001, 105, 10691.

[23] Oosawa, F.; Kasai, M. J. Mol. Biol., 1962, 4, 10.

[24] Greer, S.C. Annu. Rev. Phys. Chem., 2002, 53, 173. 
[25] Niranjan, P.S.; Forbes, J.G.; Greer, S.C.; Dudowicz, J.; Freed, K.F.; Douglas, J.F. J. Chem. Phys., 2001, 114, 10573.

[26] van Gestel, J.; van der Schoot, P.; Michels, M.A.J. Langmuir, 2003, 19, 1375.

[27] Zimm, B.H.; Bragg, J.K. J. Chem. Phys., 1959, 31, 526.

[28] Doi, M.; Edwards, S.F. The Theory of Polymer Dynamics, 10th edition, (2003); New York: Oxford Science Publications, 1986.

[29] Palmans, A.R.A.; Vekemans, J.A.J.M.; Fischer, H.; Hikmet, R.A.; Meijer, E.W. Chem. Eur. J., 1997, 3, 300.

[30] Green, M.M.; Park, J.-W.; Sato, T.; Teramoto, A.; Lifson, S.; Selinger, R.L.B.; Selinger, J.V. Angew. Chem. Int. Ed. Engl., 1999, 38, 3138.

[31] Ciferri, A. Prog. Polym. Sci., 1995, 20, 1081.

[32] Moore, J.S. Curr. Opin. Colloid Interface Sci., 1999, 4, 108.

[33] Lawrence, D.S.; Jiang, T.; Levett, M. Chem. Rev., 1995, 95, 2229.

[34] Reinhoudt, D.N.; Crego-Calama, M. Science, 2002, 295, 2403.

[35] Lehn, J.-M. Supramolecular Chemistry: Concepts and Perspectives, Weinheim: $\mathrm{VCH}, 1995$.

[36] Ben-Shaul, A.; Gelbart, W.M. In Micelles, Membranes, Microemulsions, and Monolayers, W.M. Gelbart, A. Ben-Shaul and D. Roux, Eds., New York: Springer Verlag, 1994.

[37] Ciferri, A. Ed., Supramolecular Polymers, New York: Marcel Dekker Inc, 2000.

[38] Scott, R.L. J. Phys. Chem., 1965, 69, 261.

[39] Tobolsky, A.V.; Eisenberg, A. J. Am. Chem. Soc., 1960, 82, 289.

[40] Missel, P.J.; Mazer, N.A.; Benedek, G.B.; Young, C.Y.; Carey, M.C. J. Phys. Chem., 1980, 84, 1044.

[41] Wheeler, J.C.; Pfeuty, P. Phys. Rev. A, 1981, 24, 1050.

[42] van der Schoot, P. Europhys. Lett., 1997, 39, 25.

[43] Henderson, J.R. Phys. Rev. E, 1997, 55, 5731.

[44] Schäfer, L. Phys. Rev. B, 1992, 46, 6061.

[45] Wittmer, J.P.; Milchev, A.; Cates, M.E. J. Chem. Phys., 1998, 109, 834.

[46] Wang, Z.-G.; Costas, M.E.; Gelbart, W.M. J. Phys. Chem., 1993, 97, 1237.

[47] Dudowicz, J.; Freed, K.F.; Douglas, J.F. J. Chem. Phys., 1999, 111, 7116.

[48] Cates, M.E.; Candau, S.J. J. Phys.: Condens. Matt., 1990, 2, 6869.

[49] Taylor, M.P.; Herzfeld, J. J. Phys.: Condens. Matt., 1993, 5, 2651.

[50] Odijk, T. Curr. Opin. Colloid Interface Sci., 1996, 1, 337.

[51] Cabani, S.; Paci, A.; Rizzo, V. Biopolymers, 1976, 15, 113.

[52] Qian, H.; Schellman, J.A. J. Phys. Chem., 1992, 96, 3987.

[53] Ising, E. Z. Phys., 1925, 31, 253.

[54] Kramers, H.A.; Wannier, G.H. Phys. Rev., 1941, 60, 252.

[55] Applequist, J. J. Chem. Phys., 1963, 38, 934.

[56] Palmans, A.R.A.; Vekemans, J.A.J.M.; Havinga, E.E.; Meijer, E.W. Angew. Chem. Int. Ed. Engl., 1997, 36, 2648.

[57] Brunsveld, L. private communication.

[58] Teramoto, A. Prog. Polym. Sci., 2001, 26, 667.

[59] Carlini, C.; Ciardelli, F.; Pino, P. Makromol. Chem., 1968, 119, 244.

[60] Green, M.M.; Reidy, M.P.; Johnson, R.J.; Darling, G.; O’Leary, D.J.; Willson, G. J. Am. Chem. Soc., 1989, 111, 6452.

[61] Green, M.M.; Garetz, B.A.; Munoz, B.; Chang, H.; Hoke, S.; Cooks, R.G. J. Am. Chem. Soc., 1995, 117, 4181.

[62] Goodby, J.W. J. Mater. Chem., 1991, 1, 307.

[63] Selinger, J.V.; Selinger, R.L.B. Phys. Rev. E, 1997, 55, 1728. 
[64] Selinger, J.V.; Selinger, R.L.B. Phys. Rev. Lett., 1996, 76, 58.

[65] Brunsveld, L.; Lohmeijer, B.G.G.; Vekemans, J.A.J.M.; Meijer, E.W. Chem. Commun., 2000, 2305.

[66] Brunsveld, L.; Lohmeijer, B.G.G.; Vekemans, J.A.J.M.; Meijer, E.W. J. Incl. Phenom. Macrocycl. Chem., 2001, 41, 61.

[67] van Gestel, J.; van der Schoot, P.; Michels, M.A.J. Macromolecules, 2003, 36, 6668.

[68] van Gestel, J.; van der Schoot, P.; Michels, M.A.J. J. Chem. Phys., 2004, 120, 8253.

[69] van Gestel, J. Macromolecules, 2004, 37, 3894.

[70] van Gestel, J.; Palmans, A.R.A.; Titulaer, B.; Vekremans, J.A.J.M.; Meijer, E.W. J. Am. Chem. Soc., 2005, 127, 5490. 